\title{
A Wearable Underactuated Robotic Glove Driven by Myoelectric Control Input
}

\author{
Antonio Ribas Neto*,** Matheus Del Porto* \\ Julio Fajardo*,*** Victor Ferman* Eric Rohmer* \\ * State University of Campinas, School of Electrical and Computer \\ Engineering, Campinas, São Paulo, Brazil (e-mail: \\ matheusdellporto@gmail.com; vferman1@gmail.com; \\ eric@dca.fee.unicamp.br) \\ ** Federal Institute of Education, Science and Technology Catarinense, \\ Luzerna, Santa Catarina, Brazil, (e-mail: antonio.ribas@ifc.edu.br). \\ *** Universidad Galileo, Turing Research Laboratory, FISICC, \\ Guatemala City, Guatemala (e-mail:werjevfh@galileo.edu)
}

\begin{abstract}
People who have hand impairments caused by the most common neurological and degenerative musculoskeletal diseases face trouble to achieve their everyday tasks. To try to help these people, in this works is presented a prototype of a glove-like orthosis for upper limbs. It is an underactuated robotic glove controlled by myoletric input signals collected by the Myo armband. The development and its details are all described so that it can be reproduced with improvements and used as an assistive device. To the authors' knowledge, in Brazil, there is no one similar orthosis such this one developed thus far.
\end{abstract}

Keywords: Wearable robotics glove; soft robotics; hand exoskeletons; upper limbs; assistive devices.

\section{INTRODUCTION}

The hand is fundamental to human beings develop their activities of daily living (ADLs). When disorders as stroke, cerebral paralisy, and spinal cord injury (SCI) affect the hand functioning, these hamper the accomplishment of ADLs, reducing the quality of life. In Brazil, for example, stroke is the leading cause of death and incapacity - approximately more than 100 death per 100,000 inhabitants, and among the subjects who survive the acute stroke, onethird of them remains with substantial disability (Brasil, 2013). This creates not only great social impact, since the family caregiver needs to adapt to the new routine, but also economic impact, because the majority of patients are treated in public hospitals under the national public healthcare system. Moreover, to worsen the problem, worldwide, people with disabilities have poorer health outcomes than people without disabilities, besides lower education achievements and higher rates of poverty (WHO, 2011).

To try to cope with these hindrances, over the last decade, researchers around the world have been developed a great number of soft robotic devices for helping subjects suffering from debilitating effects on hand function (Chu and Patterson, 2018; Shahid et al., 2018). Most of these wearable, or soft, robotics hand exoskeletons, are devices intended to be lightweight, portable, and affordable, mainly for providing grasping capabilities enhancement to the user. Despite the fact that most of these devices are unique, the majority of them make use of pneumatic actuators (as in Sasaki et al. (2004); Polygerinos et al. (2015)), or cable systems (for example in In et al. (2015a); Cao and Zhang (2016); Gerez et al. (2019)) as mechanism of actuation. If on the one hand pneumatic actuators can offer advantages over cable systems, for instance related to its control strategy, on the other, pneumatic actuators need to be continuously supplied with pressure to sustain the required gripping force. Alternatively, in cable systems, to keep the gripping force, it is only necessary to sustain the tension in the cable used as tendons.

Amongst the several design criteria considered in the development of orthoses, the user intent detection plays an important role, since it is used as means of control input to operate the orthosis. Commonly, the user intent detection employed in orthoses includes control input from surface electromyography (sEMG) (Polygerinos et al. (2015); Cao and Zhang (2016); Gerez et al. (2019)), optical force myography (FMG) (Fajardo et al. (2019); Ribas Neto et al. (2019)), key/button press mechanisms (Kang et al. (2016)), and wrist flexion detection by bend sensors (In et al. (2015a)), among others. According to Chu and Patterson (2018), sEMG is the most widely used method of detecting user intention, because of its trade-off between the terms of reliable signal acquisition and ease of implementation. However, the sEMG signals need to be processed, rectified, and filtered to used to control.

As a means of avoiding the signal processing some authors have used the Myo gesture control armband from Thalmic Labs (Canada), as an off-the-shelf solution. The Myo armband has been used in several works, as classification of hand motions (Mendez et al., 2017), controlling prostheses (Masson et al., 2016), orthoses (Ryser et al., 2017), and 
also for another applications (Morais et al., 2016; Hettig et al., 2015).

Taking into account the aforementioned facts, and envisioning a contribution to help these patients, in this work we present a soft robotic glove, actuated by a tendon system and driven using myoeletrical signal recording by the Myo armband. Future enhancements in this device can allow it to be used to perform the ADLs, improving the quality of life of the subjects who suffer with hand impairments. In order to present this work, this paper is organized as follows: this section introduced some of the main illness that causes hand impairments, pointed out a few works already produced upon orthoses and it was commented some points concerning the design of orthoses. In Section 2 is described the development of the soft robotic glove and its details. Some experimental results using the soft glove developed are reported in Section 3, followed by Section 4 related to conclusions and future works.

\section{MATERIALS AND METHODS}

In what follows, it is described the design of the glove and its details.

\subsection{System Architecture}

In this work, we developed a prototype of an assistive device for patients who have any degenerative illness that prevent them from provide force on fingers or have weak grasp in upper limbs when accomplishing ADLs. This device makes use of an underactuated wearable soft robotic glove controlled by the user intent detection, via the Myo gesture control armband (see Figure 1. To control the glove, the muscles activity signals provided by the Myo are sent to a personal computer (PC) through a bluetooth low-energy connection. The data are applied in a developed software routine into MatLab, using MyoMex, a package developed in MatLab code to access data from Myo. The flexion and extension movements of the soft glove fingers are operated by two Dynamixel AX-12A+ Smart Servos. Each motor is coupled to a tendon actuator mechanism. As motors rotate, they transmit forces to the glove's fingers making use of a Bowden cable/tendon system. Finally, the motor control signals are sent from PC to the motors using the U2D2 device, a small size USB communication converter that enables to control and operate the motors via the PC. Additional details on the operation of the glove are given in the following sessions.

\subsection{Glove Design}

The choice of the glove for this project is crucial. Besides being flexible, it needs to be comfortable, lightweight, and it has to be strong enough to withstand the tensile forces generated by the tendons used to actuate the fingers. Incidentally, unlike the rigid structure of conventional robots, soft wearable robots can be easily deformed when it is exerted forces on them. Some types of gloves, including Kevlar, polyester and leather gloves, as well as the combination of other fabrics, were analyzed. Based upon criteria as poor quality workmanship, type of fabric, and lack of enough pliability, a bike glove made out polyester and neoprene was selected.

In order to mimic the hand's tendon functions, little pieces of tubes were stitched in phalanges dorsal sides, distal phalanges and in the connection of the finger with the palm, to produce the necessary support for the tendons. In the first two versions of the glove developed, it was used some stopgap tubes for tests. Firstly, it was resorted to a capillary tube of domestic refrigerators, and, in the second version, to small red plastic extension straws which come with lubricating spray cans. In the two first versions employing makeshift solutions, the friction between tendons and tubes was too high, making the project unfeasible. Finally, Teflon tubes were used, a material with extremely low friction factor and resistant to abrasion. A partial view of the glove is showed in Figure 2.

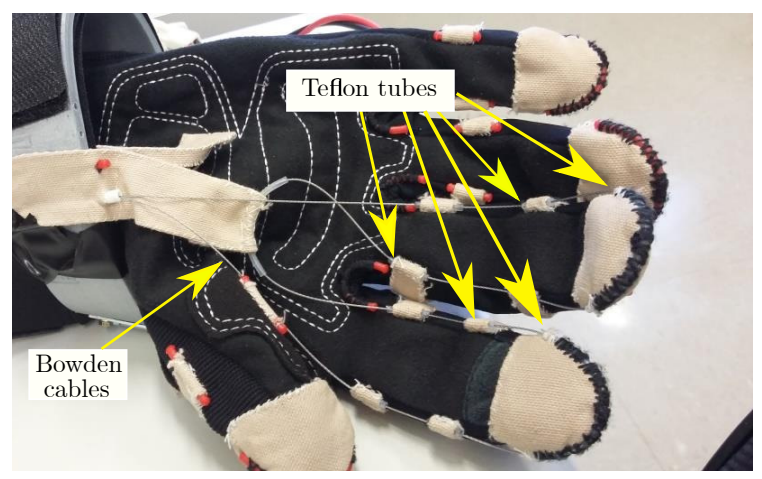

Figure 2. Glove for the project.

As the glove's fabric is flexible, thimble-like caps made from inextensible fabric were stitched at the fingertips. These act as anchor points for the tendons, providing a firm support for the U-shaped Teflon tubes rounding the fingertips. Moreover, they allow the fingers to move in the intended direction (finger extension/flexion) when tendons force the thimbles.

Another important detail here is concerned to the use of a differential mechanism. A differential mechanism evenly distributes the forces to the fingers, enabling them to adapt to different object's surfaces. Its main functions are to achieve a stable grasping and permit to reduce the number of actuators applied in the project. In soft wearable robots, it is important to try to reduce the number of actuators in order to decrease weight and cost. In the case of an underactuated soft robotic glove, it means that the extension and flexion movements of thumb, index finger and middle finger need to be achieved by using two or three motors.

\subsection{Tendons and Sheaths for the Bowden Cable System}

In the orthoses design involving cable systems, it is important to select the cable correctly because it will work similar to the human musculoskeletal system, i. e. the cable performs the tendons functions. For this application, Kevlar cable, fishing line or another flexible cable could be selected as tendons. Kevlar cables were disregard because they could not work properly with the cable winder, generating slips and consequently slacks. Hence, at first option, 


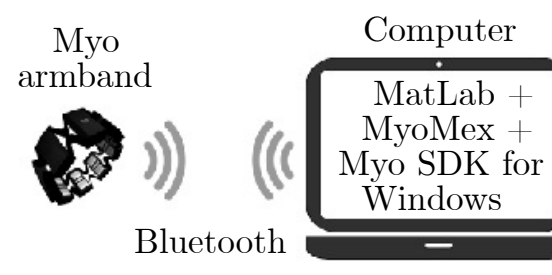

Figure 1. System architecture overview.

it was used a nylon coated fishing line from Marine Sports (0.6 $\mathrm{mm}$ in diameter) to act as tendons. It was a low-cost solution, but when conducted the tests, it was discarded due to its high stiffness. The second cable chosen was the flex-rite bead stringing wire (21 strand nylon-coated stainless steel micro-wire, $0.5 \mathrm{~mm}$ ), from Bead Smith. The conducted experiments indicated that the capacity of this cable to adjust to the spool body surface was superior when compared to that presented by the fishing line.

With respect to the Bowden cable, it is a type of flexible cable used to transmit the mechanical pulling force of an inner cable over short distances through a hollow outer cable housing (the sheath). In this project, they were used for routing the tendons and to control remotely the finger flexion and extension movements by the force exerted by the tendons. An economical and appropriate option to be used as Bowden cables are the bike brake outer casings. Pneumatic tubes made out polyurethane were also taken into consideration, but despite being cheaper, they revealed high friction when tested with the bead stringing wire. As for the two remaining sheaths seen in Figure 3, the braided one (upper) was excessively rigid and therefore difficult to bend. Thus, it was discarded and the helical (lower) was chosen.

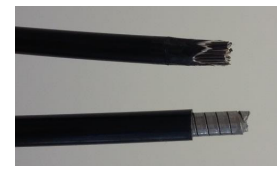

Figure 3. Details of the bike brake outer casings chosen to operate as sheaths.

\subsection{Tendon Actuator Mechanism}

In tendon-driven mechanism it is essential to keep pretension to tendons to avoid cable derailment. However, pre-tension can cause discomfort and injuries on fingers, and increase friction along the tendon route. Therefore, a slack prevention mechanism is necessary, capable of avoid pre-tension to the tendons. The device used here for actuating the tendons is a clever mechanism based on In et al. (2015b). To sum up its main characteristics, (1) this mechanism can manage in a plain manner the problems of cables derailment in similar projects involving tendon actuation; (2) making use of one-way clutches (similar to ratchet bearings), it is inherently a nonbackdrivable device, that is, it does not need energy to maintain stopped the tendons movement even when the motor responsible by turning the mechanism is shut off, what represents energy saving; (3) since it is nonbackdrivable, it applies unidirectional friction to maintain the tendon strained inside of the device while enabling slack to the tendon part out of it, this is important to avoid injuries in the hands by tension; (4) furthermore, it is a mechanism which can wind flexor tendon of each fingers set when rotating in one direction and, at the same time, unwind the extensor tendon of the same set, and vice versa. Figure 4 has a picture with the cable winder with nonbackdrivable mechanism, produced using $3 \mathrm{D}$ printing.
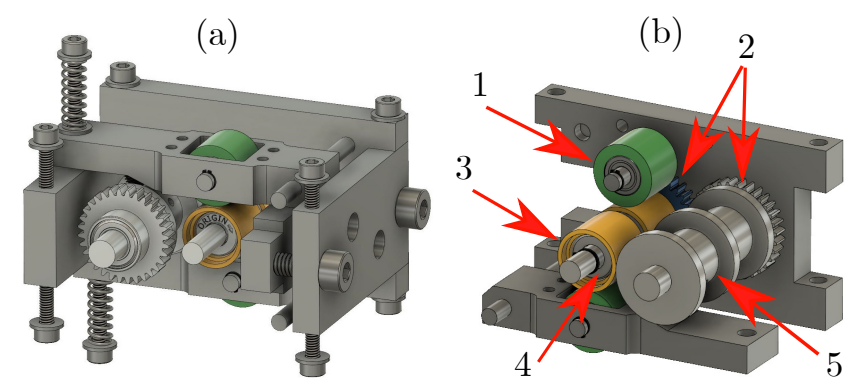

Figure 4. Cable winder with nonbackdrivable mechanism: (a) front view and (b) back view. Note: 1 - idler roller, 2 spur gears, 3 - feeder roller, 4 - one-way clutch, 5 spool.

The main purpose of the mechanism is to maintain tension to the tendon in order to prevents derailment. Its operation is the following: when the spool is rotated, it winds the tendon for flexion, for example, in one side of the spool and, at the same time releases the tendon for extension at the other side. Spool and feeder roller are coupled by a pair of spur gears, however, each feeder roller can rotates freely only in one direction, due to the assembly with the one-way clutches between the shaft and feeder roller. As the spool rotates, the one-clutch blocks the feeder roller and causes the feeder roller drag the tendon out of the mechanism. The idler roller can rotate freely in any directions, but is presses the tendon on the feeder roller and thus providing enough friction for pulling out the tendon. To warranty drag force when tendons are being released, the diameter of the spool and feeder roller, as well as the transmission between them, are designed to make the linear velocity at the surface of the feeder roller faster than the tendon unwinding speed of the spool.

\subsection{Myo Armband and Gesture Sets}

The Myo armband (see Figure 5) used in this project is a device that works collecting muscles activity via 8 units of stainless steel sEMG sensors, and arm position from an Inertial Measurement Unit (IMU). The Myo armband is worn on the user's forearm and can provide raw sEMG data related with the intended movement at a sample rate 
of $200 \mathrm{~Hz}$, and IMU data at a sample rate of $50 \mathrm{~Hz}$. The communication between Myo and computer is made via Bluetooth using an included USB bluetooth adapter, a dongle. The first time the user wears the armband it is necessary conduct a brief calibration, to armband which is made through the Myo software development kit (SDK) installed previously on the PC.

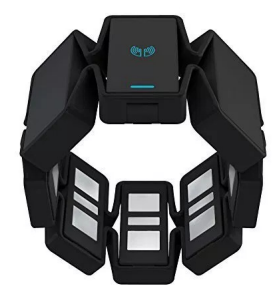

Figure 5. Myo armband.

The Myo armband promptly recognizes 6 different hand gestures, wave in, wave out, double tap, fist, spread fingers and rest gestures. In this work, it was used only three of the six gestures, wave in, wave out and spread fingers, as shown in Figure 6. The use of this gestures are associated with the control strategy and are described in details in the next section.
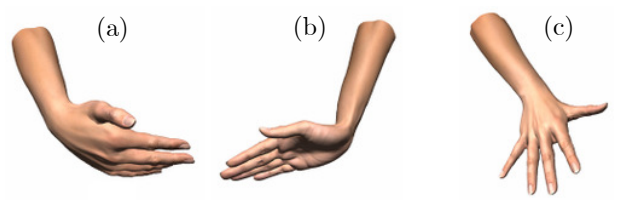

Figure 6. Hand postures used in the control loop: (a) wave in, (b) wave out, and (c) spread fingers.

To have easily access the data from the Myo, it was used the simplified m-code class called MyoMex (Tomaszewski, 2016). MyoMex is a package that enables to stream data from Myo at up to $50 \mathrm{~Hz}$ (IMU) and $200 \mathrm{~Hz}$ (EMG and meta data) into MatLab environment and Simulink.

\subsection{Control Strategy}

The control strategy was implemented to be as simple as possible. Using the Myo armband, the muscles activity is continuously measured and used to close, open, or hold the glove position, considering the user's intention. The Myo armband detects the muscles activity related to the intention of flexion or extension of the wrist, and the states are deduced from the signals acquired using the MyoMex package, which in turn, runs into MatLab. With the states extracted, the strategy of control was designed using a state machine of three states as shown in Figure 7.

The first state, "State $\neq 1$ ", is associated with the "Spread Fingers Gesture", the second and third states, "State $=2$ " and "State $=3$ ", are associated with "Wave in Gesture" and "Wave out Gesture", respectively, which in turn are linked with the extension and flexion movements of the user's wrist. The algorithm takes into account the three states as follows: while the initial condition "State $\neq 1$ " is true, the algorithm allows opening or closing the hand. When the user develops a wave in gesture, that is, a wrist flexion intention, related to "State $=2$ ", the motors responsible for closing the hand are turned on, and the soft glove starts to close. They are kept closing as long as the user remains this intention. If the user ceases the intention, the motors are turned off and the glove remains the position. If the user develops a wave out gesture (wrist extension intention), which corresponds to "State $=3$ ", the soft glove starts to open by changing the direction of the motors. It is kept opening whilst the user remains this intention. Again, when the user ceases the intention, the motors are turned off and the glove holds its position. Thus the algorithm remains in a while loop. The glove continues in this state until a new intention of the user for closing or opening the hand be detected by the Myo armband. When user executes a spread fingers gesture, the code exits the loop and then it is necessary reinitialize the algorithm in MatLab.

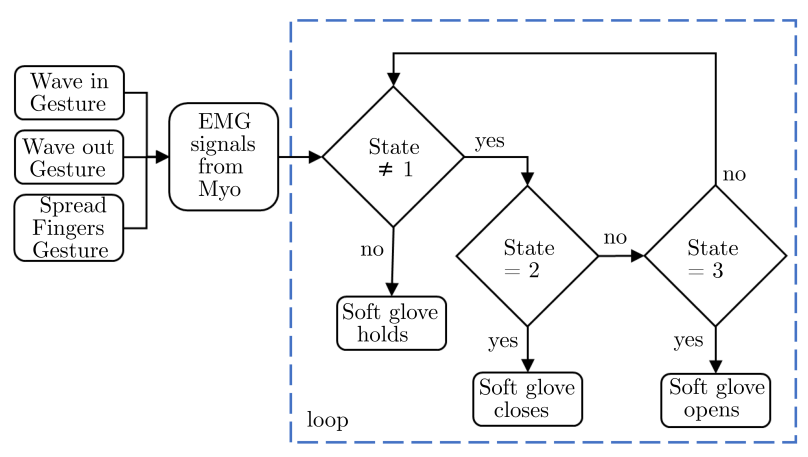

Figure 7. Main thread flowchart showing the logic control of the system. Note: Wave in Gesture = Extension Movement; Wave out Gesture = Flexion Movement.

\section{RESULTS}

Figure 8 has an image of the soft glove developed. In order to give support to the Bowden cables, one end of the sheaths are fixed to the actuator side and the other end is anchored in a customized, 3D-printed armband support adapted to the shape of the hand, which restrains the sheaths from moving toward the fingers when motors rotate. Velcro straps were used to firmly fasten the armband around the hand.

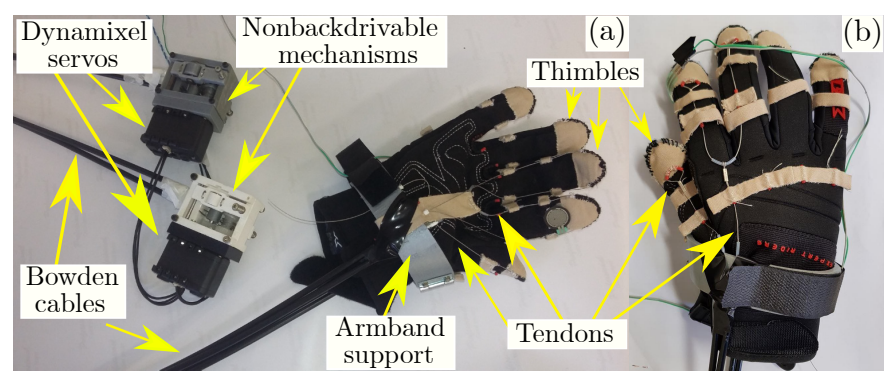

Figure 8. (a) Glove-like orthosis and its components; (b) back of the glove.

Two sets of fingers were chosen to be actuated: (1) index finger and middle finger and (2) thumb, since with this configuration is possible to perform most types of graspings commonly used to execute daily tasks. 
To qualitatively assess the glove performance, as well as to validate the control strategy, a healthy volunteer carried out some experiments. Three objects with common shapes found in everyday life were used (insulating tape, stick glue, and water bottle). The satisfactory results are shown in Figure 9.

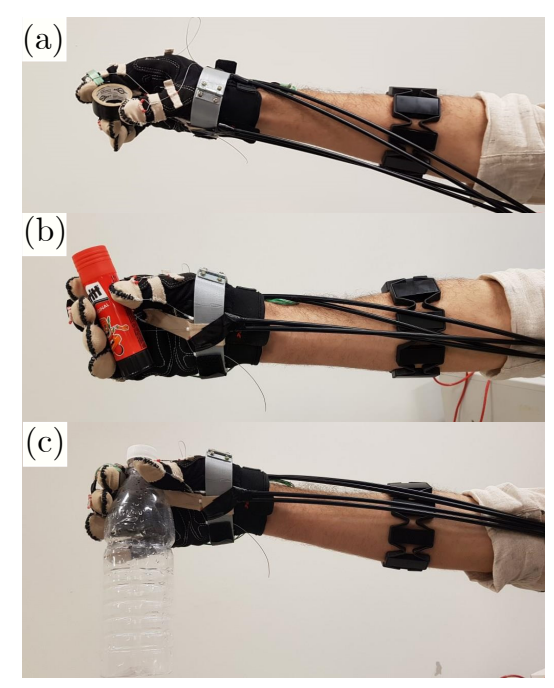

Figure 9. Objects used in the tests for hand grasping using the glove: (a) insulating tape; (b) stick glue; (c) water bottle.

As can be seen, the implemented differential mechanism allows the actuated fingers to easily adapt to the objects surface, as was expected. Moreover, it is worth to mention that as a result of using the differential mechanism, it is possible to execute the pinch grasp motion.

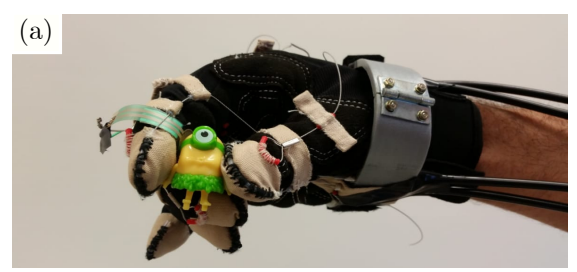

(b) Wave in gesture signal (EMG Flexion movement)
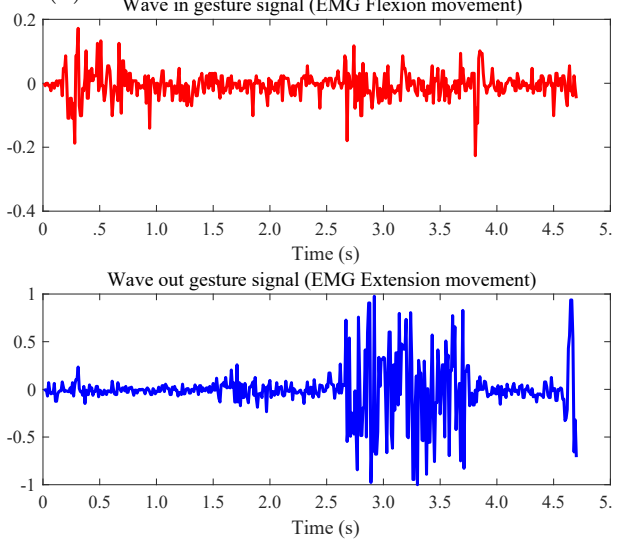

Figure 10. (a) A little toy used for testing the pinch grasp, and (b) EMG signal recorded from Wave in and Wave out gestures.
Figure 10 has an example of how the logic control of the system represented in Figure 7 works, with the EMG signals recorded associated with the Wave in and Wave out gestures. Approximately between the time window 0 and $1.5 \mathrm{~s}$ (see Fig. 10 (b) upper), the Wave in Gesture (wrist flexion) is performed, closing the hand. Notice that there is a slight variation in the EMG signal associated with the EMG extension movement (see Fig. 10 (b) lower). Between 1.5 and $2.0 \mathrm{~s}$, the toy is kept grasped and there is a small variation in both signal. Between the time window 2.5 and $4.0 \mathrm{~s}$, it is performed the Wave out Gesture (wrist extension), opening the hand. The suddenly variation after the time $4.5 \mathrm{~s}$ is related with the spread fingers gesture.

\subsection{List of Materials used in the Project}

The materials and components used in this project are listed in Table 1, with their respectively prices. It is worth commenting here that the Myo armband, the motors and the U2D2 converter were not bought specifically for this project, they are components reused from other projects. Furthermore, they cannot be promptly applied to a portable device.

Table 1. List of Material and Components used in the Project.

\begin{tabular}{cccc} 
Item & Component & Unit Price & Total $(\mathrm{R} \$)$ \\
\hline 1 & Glove (a pair) & $\mathrm{R} \$ 47.5$ & 47.5 \\
2 & Cables (10 m) & $\mathrm{R} \$ 9.36$ & 93.6 \\
3 & Sheaths (6 m) & $\mathrm{R} \$ 4.0$ & 24.0 \\
4 & One-way clutches (2 un.) & $\mathrm{R} \$ 14.00$ & $\mathrm{R} \$ 28.00^{*}$ \\
6 & Bearings (8 un.) & $\mathrm{R} \$ 15.65$ & 125.2 \\
7 & Teflon tubes (0.25 m) & $\mathrm{R} \$ 4.0 / \mathrm{m}$ & $\mathrm{R} \$ 1.0^{*}$ \\
8 & Motors (2 un.) & $\mathrm{US} \$ 44.9$ & 332.26 \\
9 & Myo armband (1 un.) & $\mathrm{US} \$ 200.0$ & 740.0 \\
10 & U2D2 (1 un.) & - & 184.63 \\
11 & Screws (several) $\$ 49.9$ & 60.0 \\
12 & Circlips (several) & - & 60.0 \\
13 & Plastic for printing (0.06 kg) & $\mathrm{R} \$ 70.0 / \mathrm{kg}$ & 4.2 \\
14 & Hinge (1 un.) & $\mathrm{R} \$ 1.5$ & 1.5 \\
15 & Velcro straps (1 m) & $\mathrm{R} \$ 6.0$ & 6.0 \\
\hline \multicolumn{4}{c}{1707.89} \\
\hline
\end{tabular}

Note: The value used to current exchange rate was US\$ $1.00=\mathrm{R} \$$ 3.70. * These components were donated.

\section{CONCLUSION AND FUTURE WORKS}

In this paper we presented a soft robotic glove controlled by myoelectric signal. The glove has the possibility to be used by people with hand dysfunction and help them to perform daily tasks. With a single actuator mechanism is possible to execute both flexion and extension movements of a set of fingers, and thus reduce the number of motors used in the project. The whole system performed as expected.

Some relevant comments are necessary. The sheaths of bicycle brake cables worked very well, but they are too tough and difficult to be bent, which makes assembly difficult. Thus, it is necessary to find a more bendable replacement for this type of sheath. Relating to the use of sEMG signals, a disadvantage is that it requires some residual level of muscle activity from users. Therefore, for a proof-of-concept, we aim to conduct some tests with stroke survivors wearing the glove for evaluation. 
Some future works related with the glove design are: (1) carry out the measurements involving the weight of the glove and the force developed by the actuated fingers set; (2) reproduce the entire glove using a more hygienic material, like as silicone, and (3) select other motors and batteries and improve software to embed the project, and thereby allowing a portable device.

\section{ACKNOWLEDGEMENTS}

The authors would like to thank Plastifluor for donating the Teflon tubes used in this project, and Origin Electric America Co., Ltd. for the one-way clutches.

\section{REFERENCES}

Brasil. Ministério da Saúde. Secretaria de Atenção à Saúde. Departamento de Ações Programáticas Estratégicas (2013). Diretrizes de atenção à reabilitação da pessoa com acidente vascular cerebral. Ministério da Saúde, Brasília (Brasil). Available in: http://bvsms.saude.gov.br/bvs/publicacoes/ diretrizes_atencao_reabilitacao_acidente_ vascular_cerebral.pdf. Last accessed: 02 May 2019.

Cao, H. and Zhang, D. (2016). Soft robotic glove with integrated sEMG sensing for disabled people with hand paralysis. In 2016 IEEE International Conference on Robotics and Biomimetics (ROBIO), 714-718. IEEE.

Chu, C.Y. and Patterson, R.M. (2018). Soft robotic devices for hand rehabilitation and assistance: A narrative review. Journal of neuroengineering and rehabilitation, 15(1), 9

Fajardo, J., Ribas Neto, A., Silva, W., Gomes, M., Fujiwara, E., and Rohmer, E. (2019). A wearable robotic glove based on optical FMG driven controller. In 2019 International Conference on Advanced Robotics and Mechatronics (ICARM). IEEE. Paper accepted to presentation on Conference.

Gerez, L., Chen, J., and Liarokapis, M. (2019). On the development of adaptive, tendon-driven, wearable exogloves for grasping capabilities enhancement. IEEE Robotics and Automation Letters, 4(2), 422-429.

Hettig, J., Mewes, A., Riabikin, O., Skalej, M., Preim, B., and Hansen, C. (2015). Exploration of 3D medical image data for interventional radiology using myoelectric gesture control. In Proceedings of the Eurographics Workshop on Visual Computing for Biology and Medicine, 177-185. Eurographics Association.

In, H., Kang, B.B., Sin, M., and Cho, K.J. (2015a). Exo-Glove: A wearable robot for the hand with a soft tendon routing system. IEEE Robotics \& Automation Magazine, 22(1), 97-105.

In, H., Lee, H., Jeong, U., Kang, B.B., and Cho, K.J. (2015b). Feasibility study of a slack enabling actuator for actuating tendon-driven soft wearable robot without pretension. In 2015 IEEE International Conference on Robotics and Automation (ICRA), 1229-1234. IEEE.

Kang, B.B., Lee, H., In, H., Jeong, U., Chung, J., and Cho, K.J. (2016). Development of a polymer-based tendon-driven wearable robotic hand. In 2016 IEEE International Conference on Robotics and Automation (ICRA), 3750-3755. IEEE.

Masson, S., Fortuna, F., Moura, F., and Soriano, D. (2016). Integrating Myo armband for the control of myoelectric upper limb prosthesis. In Proceedings of the XXV Congresso Brasileiro de Engenharia Biomédica.

Mendez, I., Hansen, B.W., Grabow, C.M., Smedegaard, E.J.L., Skogberg, N.B., Uth, X.J., Bruhn, A., Geng, B., and Kamavuako, E.N. (2017). Evaluation of the Myo armband for the classification of hand motions. In 2017 International Conference on Rehabilitation Robotics (ICORR), 1211-1214. IEEE.

Morais, G.D., Neves, L.C., Masiero, A.A., and de Castro, M.C.F. (2016). Application of myo armband system to control a robot interface. In BIOSIGNALS, 227-231.

Polygerinos, P., Galloway, K.C., Sanan, S., Herman, M., and Walsh, C.J. (2015). EMG controlled soft robotic glove for assistance during activities of daily living. In 2015 IEEE international conference on rehabilitation robotics (ICORR), 55-60. IEEE.

Ribas Neto, A., Fajardo, J., Ferman, V., Fujiwara, E., and Rohmer, E. (2019). A hybrid control strategy for tendon-actuated robotic glove and functional electrical stimulation - a preliminary study. In 2019 International Conference on Advanced Robotics and Mechatronics (ICARM). IEEE. Paper accepted to presentation on Conference.

Ryser, F., Bützer, T., Held, J.P., Lambercy, O., and Gassert, R. (2017). Fully embedded myoelectric control for a wearable robotic hand orthosis. In 2017 International Conference on Rehabilitation Robotics (ICORR), 615-621. IEEE.

Sasaki, D., Noritsugu, T., Takaiwa, M., and Yamamoto, H. (2004). Wearable power assist device for hand grasping using pneumatic artificial rubber muscle. In 2004 IEEE International Workshop on Robot and Human Interactive Communication, volume 1, 655-660. IEEE.

Shahid, T., Gouwanda, D., Nurzaman, S., et al. (2018). Moving toward soft robotics: A decade review of the design of hand exoskeletons. Biomimetics, 3(3), 17.

Tomaszewski, M. (2016). Myo SDK MATLAB MEX Wrapper, The MathWorks, Inc. https://www. mathworks.com/matlabcentral/fileexchange/ 55817-myo-sdk-matlab-mex-wrapper. Last accessed 28 April 2019.

World Health Organization (WHO) (2011). World report on disability. World Health Organization (WHO) Press, Geneva (Switzerland). Available in: https://www.who.int/disabilities/world_ report/2011/report/en/. Last accessed: 15 April 2019. 